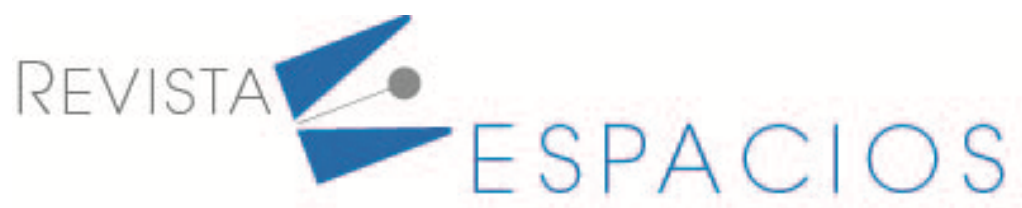

\title{
Desarrollo del pensamiento aleatorio a través de situaciones problema del contexto
}

\section{Development of random thinking through problem situations of the contextle}

\author{
PEÑA, Laura G. ${ }^{1}$ \\ CRISTANCHO, Diana C. ${ }^{2}$ \\ ESPÍNDOLA, Jorge $\mathrm{E}^{3}$
}

\begin{abstract}
Resumen
Este trabajo se inclina en fortalecer el desarrollo del pensamiento aleatorio de estudiantes de grado octavo del Instituto Educativo Técnico Diversificado de Monterrey, aplicando actividades basadas en situaciones problema del contexto. Con un enfoque mixto y un diseño de investigación acción educativa, se midió el nivel de razonamiento y pensamiento estadístico a través de dos pruebas. El análisis mediante la fusión de los niveles de Curcio y la jerarquía de Aoyama, permitió superar los niveles y potencializar su razonamiento.
\end{abstract}

Palabras clave: razonamiento estadístico, pensamiento estadístico, situaciones problema del contexto.

\begin{abstract}
This work aims to strengthen the development of random thinking in eighth grade students of the Instituto Educativo Técnico Diversificado de Monterrey, applying activities based on context problem situations. Following a mixed approach and an educational action research design, the level of reasoning and statistical thinking was measured through two tests. The analysis was done based on merging Curcio's levels and Aoyama's hierarchy, this one allowed to overcome the levels and promote its reasoning.

keywords: statistical reasoning, statistical thinking, context problem situations.
\end{abstract}

\section{Introducción}

La cultura estadística es considerada como parte de las competencias que hoy debe poseer un ciudadano del mundo (NCTM, 2000). Es importante que los estudiantes, desarrollen la capacidad de lectura de tablas y gráficos estadísticos, así mismo logren realizar predicciones e inferencias sobre situaciones del contexto, es decir, posean un nivel de razonamiento y pensamiento estadístico que les permita realizar acciones básicas como: interpretar y evaluar críticamente la información publicada diariamente en medios de comunicación, audiovisuales y escritos.

\footnotetext{
${ }^{1}$ M.Sc. Universidad Pedagógica y Tecnológica de Colombia. Escuela de Licenciatura en Matemáticas. PIRÁMIDE. Colombia. Email: lauragivelly.pena@uptc.edu.co

${ }^{2}$ M.Sc. Instituto Educativo Técnico Diversificado de Monterrey. Colombia. Email: aponte_9@hotmail.com

${ }^{3}$ Ph.D. Universidad Pedagógica y Tecnológica de Colombia. Escuela de Ingeniería de Sistemas y Computación. INFELCOM. Colombia. Email: jespindola@uptc.edu.co
} 
Según Zapata y Rocha (2016), para promover el desarrollo del pensamiento estadístico se necesita mucho más que una enseñanza de tipo informativa en la que el profesor transmite y explica el saber. Es por esto que, como nueva generación de docentes, se tiene la obligación y el deber de buscar nuevas estrategias que permitan al estudiante entender, pensar, analizar y razonar sobre situaciones o problemas que surgen en el diario vivir y que a la vez, generan incertidumbre y preocupación. La estadística es una herramienta fundamental para analizar y entender los problemas en un mundo tan complejo como el actual (Galli, 2015).

Para Zapata y Rocha (2016), el pensamiento estadístico enfatiza:

El desarrollo de una visión crítica y las habilidades para la resolución de problemas reales dentro de un contexto particular en la cual la cuantificación y reducción de la variación son esenciales. Incluye el dominio de conceptos y procedimientos, construcción de modelos, razonamiento, inferencia, desarrollo de disposiciones pero no aislado sino en relación a un proceso. (p. 74)

Estudios recientes han revelado que los profesores en el aula de clase, se enfocan en realizar preguntas más orientadas a estimular el conocimiento y procedimiento, en ausencia de contextos reales, que en un razonamiento estadístico de orden superior (Zapata \& Rocha, 2016).

El proceso de estudio del pensamiento aleatorio y situaciones problema en contexto, ha ocupado a los investigadores en reuniones, congresos y otros eventos, tales como: la Conference of European Research in Mathematics Education (CERME), la Conference of the International Group for the Psychology of Mathematics Education (PME), el International Congress on Mathematical Education (ICME), las Reuniones Latinoamericanas de Matemática Educativa (RELME), los Encuentros Colombianos de Matemática Educativa (ECME), entre otros. Por ejemplo, en el ICME 14 se presenta el Topic Study Group number 12 (TSG 12) que se dirige a buscar contribuciones en la investigación y el desarrollo de la estadística en la enseñanza y aprendizaje como la "ciencia de los datos" en todos los niveles educativos. En este se mencionan trabajos académicos que incluyen tendencias globales, desarrollo y evaluación de la alfabetización estadística, incluida la conexión de la educación estadística, con las cuestiones sociales y políticas, preparación y entornos de aprendizaje ricos en tecnología.

En Colombia, el Ministerio de Educación Nacional (MEN) plantea a través de las competencias matemáticas, lo que los estudiantes deben saber y saber hacer con el conocimiento que ellos adquieren en la Educación Básica y Media (MEN, 2006). A partir de lo anterior, se observa la importancia que el pensamiento estadístico y aleatorio tienen en el desarrollo y aprestamiento de las competencias que debe tener todo estudiante al finalizar el bachillerato, las cuales son evaluadas en las pruebas de estado realizadas por el Instituto Colombiano para el Fomento de la Educación Superior (ICFES). Los Lineamientos Curriculares del Ministerio de Educación Nacional hacen referencia al pensamiento aleatorio (MEN, 1998), así también, los Estándares Curriculares de Matemáticas especifican los propósitos del mismo (MEN, 2006).

En la actualidad, la estadística hace parte de los planes de estudio de matemáticas en Educación Básica y Media, pero como lo expone Agnelli (2009) citado por Osorio, Suarez y Uribe (2011, p. 363) no se le da la importancia que merece y aunque son varios los trabajos de investigación realizados sobre didáctica de la probabilidad, como se evidencia en los boletines publicados por la International Association for Statistical Education (IASE) no es suficiente, es decir, que aunque existe una preocupación por realizar y divulgar trabajos de investigación que evidencien la realidad de la didáctica de la probabilidad, así como algunas estrategias que permiten alcanzar los objetivos propuestos y que motiven al docente a la elaboración de materiales que sigan apoyando tal aprendizaje, los avances son todavía mínimos, debido a que el diagnóstico de la situación no es suficiente para enfrentar esta problemática.

Sumado a esta panorámica, gran parte de los docentes nunca han recibido una educación formal en estadística. En ese sentido, Zapata y González (2017) afirman que "en Colombia los profesores han sido formados en programas profesionales de educación matemática con un número importante de cursos para apoyar el 
componente numérico y variacional, pero un limitado número de cursos para el componente aleatorio" (p. 63). Cabe mencionar que en el campo de la estadística y la matemática se le da prioridad a enseñar algoritmos que, finalmente, son muy fáciles de obtener mediante una calculadora, lo realmente importante, es enseñar una cultura estadística como lo plantea Gal (2002), en la cual la variación y el contexto real son elementos esenciales del aprendizaje significativo.

\subsection{Fundamentación del problema}

Según la página del gobierno de Colombia, para el año 2019, el departamento del Casanare obtuvo en las pruebas Saber 11 un puntaje promedio de 247,07, ocupando así el décimo puesto a nivel nacional; esta situación es preocupante, ya que si bien es cierto que no se encuentra en los últimos lugares, son en su mayoría los colegios privados quienes obtienen puntajes altos, logrando así posicionar al departamento. Ahora bien, los resultados ICFES obtenidos en el año 2019 por desempeño en el área de matemáticas en el municipio de Monterrey, preocupan aún más, pues al realizar el contraste con los demás municipios, éste obtuvo 2,5 en una escala de 0 4. Dichos resultados ilustran que se debe fortalecer los componentes y competencias que tiene establecido el ICFES al momento de evaluar.

Entre los componentes que evalúa el ICFES en las pruebas de estado, como en las pruebas SABER 3ㅇ, 5ㅇ y 9오 se encuentra el componente aleatorio el cual:

Corresponde a la representación, lectura e interpretación de datos en contexto; el análisis de diversas formas de representación de información numérica, el análisis cualitativo de regularidades, de tendencias, y la formulación de inferencias y argumentos usando medidas de tendencia central y de dispersión; y por el reconocimiento, descripción y análisis de eventos aleatorios. (ICFES, 2017, p. 37)

También se evalúan competencias matemáticas que se encuentran agrupadas de la siguiente manera: razonamiento y argumentación; planteamiento y resolución de problemas; comunicación, representación y modelación; esta última, integrada con el componente aleatorio, se propone una serie de criterios que los estudiantes de 8 ㅇ a 9을 grado deben superar satisfactoriamente como lo son: la capacidad para expresar ideas, describir relaciones matemáticas, comparar e interpretar datos de situaciones reales, hallar la media de un conjunto de datos, entre otros.

Con las consideraciones anteriores, y la aplicación de métodos empíricos como entrevistas y encuestas a docentes del área, y la experiencia de los investigadores, se pudo constatar que existen algunas dificultades como:

- Son insuficientes los conceptos básicos que tienen los estudiantes en estadística para lograr cumplir con los Derechos Básicos de Aprendizaje (DBA) propuesto para grado 8‥

- Presentan apatía por la materia, ya que los docentes utilizan una intensidad horaria mayor en el área de Geometría que en Estadística.

- Los docentes que orientaron la asignatura fueron cambiados en varias oportunidades, trayendo consigo cambios de metodología.

Las valoraciones anteriores y el estudio epistemológico inicial realizado, permiten determinar el siguiente problema de investigación: ¿Cómo favorecer el desarrollo del pensamiento aleatorio, a través de actividades enfocadas en situaciones problema del contexto, en estudiantes de grado octavo del Instituto Educativo Técnico Diversificado de Monterrey? 


\subsection{Objetivos e hipótesis científica}

Se precisa como objetivo general, propiciar el desarrollo del pensamiento aleatorio en un grupo de estudiantes de grado $8^{\circ}$ de este Instituto a partir de situaciones problema del contexto. Para la consecución del objetivo y la solución del problema, se presenta la siguiente hipótesis científica: la aplicación de actividades basadas en situaciones problema del contexto, como método de enseñanza, contribuyen en mayor grado al desarrollo del razonamiento y pensamiento estadístico.

En aras de dar cumplimiento al objetivo, y lograr resolver el problema planteado, así como para guiar el curso de la investigación, se inició un estudio mixto, con la aplicación del proceso metodológico de investigación, acción educativa, con las siguientes tareas de investigación:

- Determinar el nivel de razonamiento y pensamiento aleatorio que tienen los estudiantes de grado $8^{\circ}$ a partir de la aplicación de una prueba diagnóstica.

- Diseñar actividades basadas en el contexto de los estudiantes, con distintos niveles de complejidad, según el avance observado.

- Implementar actividades de profundización enfocadas en situaciones problema del contexto que permitan potencializar el desarrollo del pensamiento aleatorio.

- Analizar los avances o falencias, en cuanto al nivel de razonamiento y pensamiento aleatorio de los estudiantes, con la aplicación de una prueba diagnóstica final.

\section{Estado del Arte}

Se presentan algunas investigaciones a nivel local, nacional, e internacional cuyos trabajos por sus características similares aportan a este trabajo, pero también evidencian que se presenta una investigación innovadora en lo referente al el desarrollo del pensamiento aleatorio.

A nivel local, se tiene el trabajo de Palacios y Tafur (2018) quienes desarrollaron una tesis de maestría en la Universidad de La Salle, titulada "La resolución de problemas como estrategia de enseñanza para el desarrollo del pensamiento aleatorio". Este es un estudio realizado con estudiantes de grado quinto y sexto de dos instituciones de Yopal Casanare, se plantearon como objetivo general describir la manera en que el proceso de resolución de problemas, como estrategia de enseñanza, favorece el desarrollo de pensamiento aleatorio en estudiantes de dos instituciones educativas.

A nivel nacional, en el año 2014, García, Hoyos y Acosta en su trabajo titulado "El desarrollo del pensamiento estadístico en niños y niñas del grado $7^{\circ}$ de básica secundaria, una estrategia guiada por software educativo", plantearon como objetivo establecer experimentalmente si la aplicación de un software educativo contribuye a la consolidación del pensamiento estadístico en un grupo de estudiantes de grado $7^{\circ}$. Para esto diseñaron y aplicaron un pre-test y pos-test, con los que lograron comparar resultados y llegar a conclusiones relevantes.

Por otra parte, González (2018) realiza una investigación titulada "Resolución de problemas, una estrategia para aprender estadística", para este proyecto de investigación plantea como objetivo general identificar los cambios que se evidencian en la comprensión de datos representados en tablas y gráficos estadísticos, al desarrollar una propuesta didáctica centrada en la resolución de problemas matemáticos, con estudiantes del grado $5^{\circ}$ de la sede rural El Rhur de la Institución Educativa Divino Salvador de Cucunubá.

Para el año 2017, Ramírez realizó una investigación titulada "Fortalecimiento del pensamiento aleatorio en estudiantes de grado tercero de la Institución Educativa Académica de Guadalajara de Buga en el segundo semestre de 2016", donde planteó como objetivo general fortalecer el pensamiento aleatorio y los sistemas de datos en un grupo de estudiantes de grado $3^{\circ}$ de primaria. 
A nivel Internacional, en el año 2002, Chick y Watson realizaron un trabajo de investigación titulado "Collaborative Influences on Emergent Statistical Thinking - a case Study". El propósito de esta investigación fue examinar cómo el trabajo colaborativo de un grupo de tres niños de grado $6^{\circ}$ influye en el pensamiento estadístico presente en cada uno de ellos.

Por otra parte, en el año 2011, Jiménez e Insunza presentaron en la XIII Conferencia Interamericana de Educación Matemática su trabajo de investigación titulado "Razonamiento y Pensamiento Estadístico en estudiantes Universitarios" de la Universidad Autónoma de Sinaloa. Ellos realizaron un estudio sobre la caracterización del nivel de desarrollo del razonamiento y pensamiento estadístico en estudiantes con preparación matemática avanzada: el caso de las pruebas de hipótesis, usando el modelo de "Estructura del Proceso de Aprendizaje Observado" Structure of Observed Learning Outcomes (SOLO por sus siglas en inglés) propuesto por Biggs y Collis en 1982.

\section{Referentes teóricos}

Los referentes teóricos pertinentes para el desarrollo de esta investigación, están dados en: estocástica y su didáctica para maestros y los niveles de comprensión de la información contenida en tablas y gráficos. Además, de establecer los conceptos de: competencia estadística, razonamiento estadístico, pensamiento estadístico y situación problema.

\subsection{Estocástica y su didáctica para maestros}

La elaboración de tablas y datos no es una tarea sencilla, pero son muchos los docentes que piensan que sí y dedican poco tiempo a su enseñanza. Según Batanero y Godino (2002):

Elaborar una tabla de frecuencias o un gráfico supone ya una primera reducción estadística, pues se pierden los valores originales de cada uno de los datos individuales pasándose a la distribución de frecuencias. Este concepto es ya complejo, al referirse al conjunto de los datos y no a cada caso particular. Mientras que los niños comprenden bien propiedades que se refieren a individuos, como el color de pelo de una persona o su altura, les resulta más problemático comprender la idea de distribución del color de pelo de un grupo. La destreza en la lectura crítica de datos es una necesidad en nuestra sociedad tecnológica, ya que se encuentran tablas y gráficos en la prensa, comercio, así como en distintas asignaturas del currículo. (p. 726)

\subsection{Niveles de comprensión de la información contenida en tablas y gráficas estadísticas}

Se pueden distinguir cuatro niveles de comprensión de gráficos propuestos por Curcio (1989) y, Friel, Curcio y Bright (2001) que pueden aplicarse a las tablas y gráficos estadísticos. El objetivo de la educación estadística sería llevar a cada alumno a adquirir el mayor nivel para el cual este capacitado:

- Lectura literal (leer los datos): este nivel de comprensión requiere una lectura literal del gráfico; no se realiza interpretación ni cálculos de la información contenida en el mismo.

- Interpretar los datos (leer dentro de los datos): incluye la interpretación e integración de los datos en el gráfico; requiere la habilidad para comparar cantidades y el uso de otros conceptos y destrezas matemáticas.

- Hacer una inferencia (leer más allá de los datos): requiere que el lector realice predicciones e inferencias a partir de los datos, sobre informaciones que no se reflejan directamente en el gráfico. Un ejemplo de este nivel, es estimar la temperatura del día próximo a los especificados en el gráfico.

- Valorar los datos (leer detrás de los datos): supone valorar la fiabilidad y completitud de los datos, como hacer un juicio sobre si realmente las preguntas de la encuesta miden la práctica de deporte, o cómo podríamos medirlo de una forma más fiable. 
Desde la Jerarquía de Aoyama (2007), se presenta en la Tabla 1, un modelo en el cual se establece una jerarquía en la comprensión de la información que realizan los estudiantes a partir de tablas y gráficas estadísticas. En cada una de ellas, se plantean las dificultades que pueden presentar en la descripción y representación de datos y que a su vez se pueden ver reflejadas cuando intercambian los ejes y sus unidades de medida; un error muy común, es omitir etiquetas para identificar las variables expresadas en las gráficas y realizar escalas diferentes en cada uno de los ejes.

Tabla 1

Modelo Jerárquico propuesto por Kazuhiro Aoyama (2007)

\begin{tabular}{|c|l|}
\hline Idiosincrático & $\begin{array}{l}\text { No pueden leer valores o tendencias en gráficas o proporcionan valores incorrectos. Fallan } \\
\text { al conectar algunas características extraídas de las gráficas con el contexto. }\end{array}$ \\
\hline Lectura básica de gráficas & $\begin{array}{l}\text { Pueden leer valores y tendencias en las gráficas, pero no pueden explicar los significados } \\
\text { contextuales de las tendencias o características que ellos observan en los datos. }\end{array}$ \\
\hline Relacional & $\begin{array}{l}\text { Los estudiantes pueden leer valores particulares y tendencias así como explicar los } \\
\text { significados contextuales en función de las características mostradas en una gráfica, pero no } \\
\text { pueden sugerir interpretaciones alternativas, usan solo los significados presentados. }\end{array}$ \\
\hline Crítico & $\begin{array}{l}\text { Pueden leer gráficas y comprender las variables contextuales presentadas; asíno, } \\
\text { pueden evaluar la fiabilidad de la información descrita en la gráfica, pero no son capaces de } \\
\text { buscar otras hipótesis. }\end{array}$ \\
\hline Hipotetizador & $\begin{array}{l}\text { En este nivel los estudiantes pueden leer gráficas, aceptar y evaluar la información } \\
\text { presentada; también pueden formar sus propias hipótesis explicativas o modelos y actuar } \\
\text { como “investigadores" estadísticos activos y no solo como receptores de información. }\end{array}$ \\
\hline
\end{tabular}

Fuente: Aoyama, K. (2007)

\subsection{La competencia estadística}

Para Watson (2006) y Gal (2004) citados en Sánchez y Gómez (2011) la competencia estadística consiste en:

Los conocimientos, habilidades y disposiciones estadísticos que todo ciudadano debe tener para funcionar adecuadamente en una sociedad caracterizada por la circulación de grandes cantidades de información, involucra la comprensión y el uso del lenguaje y las herramientas básicas de la estadística. (p. 3)

\subsection{El razonamiento estadístico}

Según (Garfield y Ben-Zvi, 2008a) citado en Sánchez et al. (2011) el razonamiento estadístico es "la manera de razonar con ideas estadísticas, es decir, consiste en realizar inferencias o deducciones y utilizarlas en la solución de problemas propios del campo. Implica conectar conceptos estadísticos y probabilísticos, entender y explicar procesos estadísticos e interpretar los resultados" (p. 3).

\subsection{El pensamiento estadístico}

Para Wild y Pfannkuch (1999) citado en Sánchez y Gómez (2011) el desarrollo del pensamiento estadístico consiste en:

La forma en que piensa un estadístico profesional. Implica saber cómo, dónde y por qué llevar a cabo una investigación estadística, así como utilizar un método, aplicar un modelo o idear un diseño estadístico; para hacerlo se requiere una comprensión profunda de las teorías que subyacen a los métodos y procesos estadísticos. (p. 3)

\subsection{Situación Problema}

Para este trabajo se aborda la definición dada por Obando y Muñera (2003) para quienes se trata de: 
Un contexto de participación colectiva para el aprendizaje, en el que los estudiantes, al interactuar entre ellos mismos, y con el profesor, a través del objeto de conocimiento, dinamizan su actividad matemática, generando procesos conducentes a la construcción de nuevos conocimientos. Así, ella debe permitir la acción, la exploración, la sistematización, la confrontación, el debate, la evaluación, la autoevaluación, la heteroevaluación. (p. 185)

\section{Metodología}

\subsection{Enfoque de Investigación}

Este trabajo se presenta desde un enfoque de investigación mixto. Para lograr comparar los resultados obtenidos en las pruebas diagnósticas inicial y final, así como, determinar si hubo un avance significativo, se utiliza el programa estadístico informático SPSS. Lo anterior evidencia el enfoque cuantitativo que según Hernández (2006) "se fundamenta en analizar una realidad objetiva a partir de mediciones numéricas y análisis estadísticos para determinar predicciones o patrones de comportamiento del fenómeno o problema planteado" (p. 5).

El enfoque cualitativo, se evidencia en la estructura de las preguntas propuestas en las pruebas diagnósticas inicial y final que fusionan los niveles de Curcio y la Jerarquía de Aoyama (véase Tabla 4); estos niveles proponen una serie de aspectos que deben ser superados en su totalidad para lograr subir de nivel.

La metodología que sigue esta propuesta de trabajo se fundamenta dentro del tipo de investigación acción educativa, la cual define Gómez (2003) como “... una aplicación de los procesos metacognitivos a la propia práctica en pos del mejoramiento del aprendizaje de sus alumnos, del mejoramiento de la profesión y de la superación del aislamiento del docente" (p. 93).

Para esta investigación, se proponen varias actividades en las cuales los estudiantes de grado octavo que se tomaron como muestra, dieron solución a situaciones problema del contexto, de manera individual y grupal. Llevando así un control y seguimiento de las dificultades y fortalezas observadas en clase, durante el desarrollo de cada una de las actividades, se logra reflexionar sobre la propia práctica docente.

\subsection{Población y muestra}

Un grupo de estudiantes del Instituto Educativo Técnico Diversificado (IETD), ubicado en el municipio de Monterrey, Casanare; esta institución es de carácter público y cuenta con aproximadamente 2100 estudiantes. Para el año académico 2019 se contaba con cinco grupos de grado $8^{\circ}$ conformados entre 25 a 30 estudiantes por salón. Para seleccionar el grupo muestra, se aplicó la prueba diagnóstica inicial a cada grupo y aunque todos presentaban falencias en cuanto al pensamiento aleatorio, se selecciona el grado que presentó más bajo nivel. Las actividades propuestas se aplicaron a 26 estudiantes de grado 8-2 del año 2019. Los estudiantes son de estrato socioeconómico 1 y 2 , cuyas edades oscilan entre 13 y 15 años.

\subsection{Métodos e Instrumentos}

La Tabla 2, presenta los métodos del nivel empírico que se utilizan en la investigación con una breve descripción. 
Tabla 2

Métodos empíricos utilizados en la investigación

\begin{tabular}{|c|l|}
\hline $\begin{array}{c}\text { Prueba diagnóstica - } \\
\text { Inicial }\end{array}$ & $\begin{array}{l}\text { Para identificar el nivel de comprensión que poseen los estudiantes al momento } \\
\text { de observar tablas y gráficos estadísticos y así determinar el nivel en el que se } \\
\text { encuentran según la jerarquía propuesta que fusiona los niveles de Friel, Curcio } \\
\text { y Bright (2001) y la jerarquía de Aoyama (2007). Además, determinar si logran } \\
\text { clasificar un suceso como probable e improbable y así hallar la probabilidad de } \\
\text { una situación del contexto planteada. }\end{array}$ \\
\hline $\begin{array}{c}\text { Actividad N²: ¿Qué } \\
\text { asignatura van perdiendo } \\
\text { los alumnos de mi grado? }\end{array}$ & $\begin{array}{l}\text { Con el fin de introducir a los estudiantes en conceptos básicos de estadística, } \\
\text { tales como población, muestra, variables y tipos de variables, al igual que tablas } \\
\text { de frecuencias y tipos de representación gráfica según la variable de estudio. } \\
\text { Todo esto a partir de ejemplos del contexto de cada uno de los estudiantes y una } \\
\text { situación problema general. }\end{array}$ \\
\hline $\begin{array}{c}\text { Actividad N²: “Conozco } \\
\text { mejor a mis compañeros” }\end{array}$ & $\begin{array}{l}\text { Orientada a realizar un análisis estadístico a partir de una encuesta de } \\
\text { caracterización que se aplicó a estudiantes de grado }{ }^{\circ} \text { y que sirvió como soporte } \\
\text { de información para la Institución Educativa, ya que involucró cuatro aspectos } \\
\text { importantes: fisiológico, socioeconómico, socio afectivo y educativo. }\end{array}$ \\
\hline $\begin{array}{l}\text { Prueba diagnóstica - Final } \\
\text { Encaminada a comparar los resultados obtenidos en la prueba diagnóstica inicial } \\
\text { yinal, tomando como guía la jerarquía propuesta que fusiona los niveles de } \\
\text { Curcio y Aoyama (véase tabla 4); y así determinar si el nivel de razonamiento } \\
\text { estadístico y pensamiento estadístico, se fortalece con la aplicación de } \\
\text { actividades basadas en situaciones problema del contexto. }\end{array}$ \\
\hline
\end{tabular}

Fuente: elaboración propia

En este orden de ideas, es importante resaltar que una vez procesados los resultados de la prueba diagnóstica inicial, final y las actividades con el apoyo del software SPSS, se precisa de la triangulación empírica para buscar las regularidades existentes y así poder establecer los resultados y conclusiones generales de la investigación.

\subsection{Fases de desarrollo}

\section{Fase 1. Diseño de prueba diagnóstica - inicial}

La prueba inicial estructurada en cinco puntos consta de los siguientes niveles:

\section{Tabla 3}

Niveles propuestos en la prueba diagnóstica - inicial

\begin{tabular}{|l|l|}
\hline \multicolumn{1}{|c|}{ Curcio (1989) } & \multicolumn{1}{c|}{ Aoyama (2007) } \\
\hline Leer los datos & Lectura básica de gráficas \\
\hline Leer dentro de los datos & Relacional \\
\hline Leer más allá de los datos & Crítico \\
\hline
\end{tabular}

Fuente: elaboración propia

\section{Fase 2. Aplicación de la prueba diagnóstica}

Para el análisis de esta segunda fase, se organiza las respuestas de los estudiantes de acuerdo con la comprensión y análisis crítico en su interpretación. Para esto, se toma como guía los niveles descritos en los referentes teóricos fusionándolos en una sola jerarquía (ver Tabla 4). 
Tabla 4

Jerarquía propuesta fusionando los Niveles

de Curcio y la Jerarquía de Aoyama

\begin{tabular}{|l|l|}
\hline Nivel de comprensión & \multicolumn{1}{c|}{ Descripción } \\
\hline Nivel 1: lectura Literal & $\begin{array}{l}\text { La lectura e interpretación del gráfico, se basa en palabras referentes al título o variables; no } \\
\text { logra realizar interpretaciones, basándose en características observadas. }\end{array}$ \\
\hline Nivel 2: comparativo & $\begin{array}{l}\text { La lectura e interpretación de gráficos, presenta comparaciones de datos, según las } \\
\text { características mostradas. No se logra sugerir interpretaciones alternativas. }\end{array}$ \\
\hline Nivel 3: predictivo & $\begin{array}{l}\text { La lectura e interpretación de gráficos, presenta predicciones del comportamiento de los datos } \\
\text { correspondientes al valor de la variable; logra establecer interpretaciones a futuro según el } \\
\text { contexto. }\end{array}$ \\
\hline Nivel 4: integrativo & $\begin{array}{l}\text { La lectura e interpretación de gráficos, integra tres aspectos: contexto, crítica e hipótesis; es } \\
\text { decir, leer gráficas, aceptar y evaluar la información presentada, formar sus propias hipótesis y } \\
\text { actuar como investigador. }\end{array}$ \\
\hline
\end{tabular}

Fuente: elaboración propia

\section{Fase 3. Diseño de las actividades de profundización}

Se aplicaron dos actividades que se enfocaron en situaciones problema del contexto; en cada actividad se aumentó la complejidad, según los avances que se fueron observando en el grupo de estudiantes.

Se inició con una situación problema reflejada dentro del aula, en la cual se hizo énfasis en la clasificación de variables cualitativas y cuantitativas, representación gráfica de los dos tipos de variables, y lograr así hacer una lectura literal de las gráficas sin realizar cálculos.

Se continuó con una situación problema que abarcó a la institución, cuyo fin era caracterizar a todos los estudiantes que cursan grado $8^{\circ}$. Para lograr el objetivo propuesto se diseñó una encuesta en la cual se obtuvo información necesaria que permitió al grupo de estudiantes realizar un análisis estadístico, en el cual fue posible comparar cantidades y sacar conclusiones del mismo. Como segunda parte de esta actividad, se trabajó con un software estadístico (Excel), teniendo en cuenta que la muestra que se tomo es grande y las preguntas realizadas en la encuesta son extensas; además, como una estrategia metodológica para motivar a los estudiantes y a la vez aprovechar que la mayoría de ellos cuentan con un celular de última tecnología que les permite descargar la aplicación y trabajar de una manera más cómoda e innovadora.

\section{Fase 4. Prueba diagnóstica - Final}

Para la elaboración, se tuvieron en cuenta tres factores importantes:

- Los gráficos y tablas fueron similares a los propuestos en la prueba inicial; esto con el fin de medir y comparar resultados.

- Con base en los resultados obtenidos en la prueba diagnóstica inicial, se eliminaron situaciones donde tenían que determinar sucesos y probabilidades, debido a que se evidenció mayor dificultad y debilidades en la lectura e interpretación de gráficos.

- En remplazo de las situaciones de probabilidad, se propuso una situación problema que busca identificar si el estudiante alcanza el nivel 4: integrativo (ver tabla 4).

\section{Análisis y resultados}

\subsection{Análisis de la Prueba diagnóstica - Inicial}

I. De las preguntas planteadas del numeral 1 al 6 , se obtuvieron los siguientes resultados: 


\section{Gráfico 1}

Nivel №1 "Lectura literal" - P. Inicial

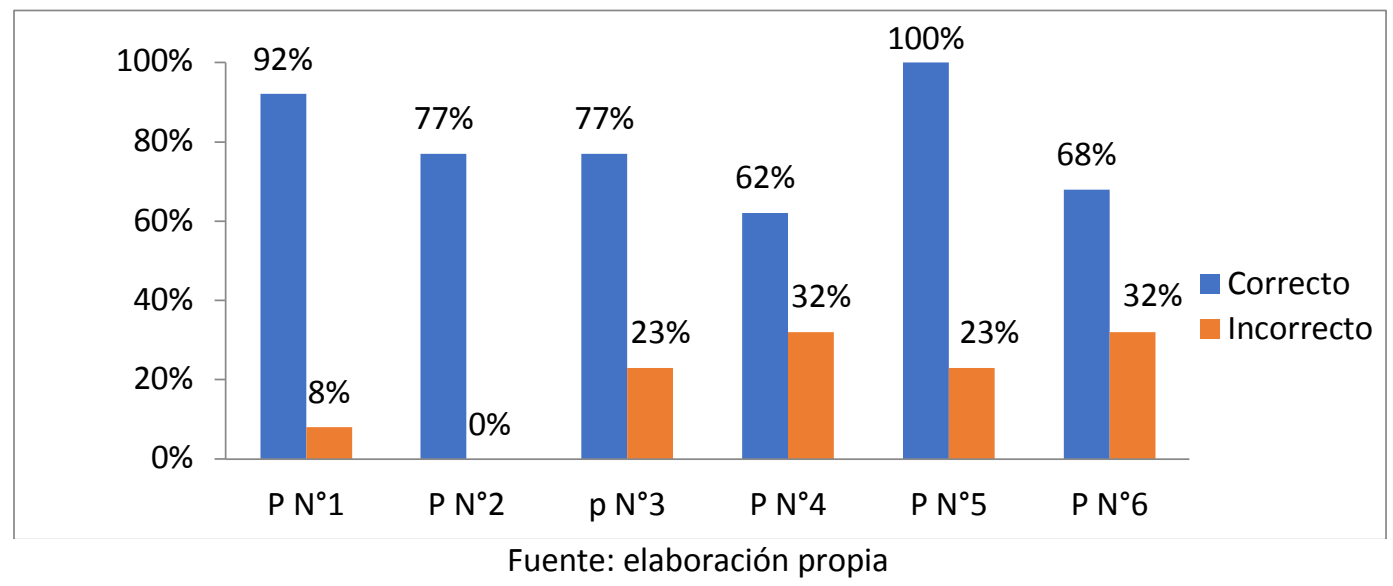

Análisis: el grupo de estudiantes supera satisfactoriamente el primer nivel de lectura literal de gráficos, es decir, logran leer los datos del gráfico sin necesidad de realizar una interpretación de la información contenida en el mismo.

II. Dada la primera situación: Andrea tiene una bolsa con dulces; 5 son de limón, 2 de fresa y 1 de menta, se obtienen los siguientes resultados:

Posibilidad de un suceso: si Andrea saca sin mirar un dulce de la bolsa ¿Es posible o imposible que el dulce sea de fresa? ¿Por qué?

Análisis: con un $77 \%$ que responden acertadamente, se puede destacar que son muy pocos los estudiantes que presentan dificultad al determinar la posibilidad de un suceso.

Sucesos predecibles: su amiga Pilar pregunta ¿es más probable sacar de la bolsa, un dulce de limón que uno de fresa? Explica por qué.

Análisis: con un $92 \%$ que responden acertadamente, se tiene que la mayoría de los estudiantes logran determinar qué suceso es más predecible que el otro.

Suceso Improbable: ¿Qué es más probable que saque, un dulce de limón o de naranja?

Análisis: todos los estudiantes respondieron correctamente a la pregunta planteada, lo que indica que identifican cuando un caso es improbable.

De la segunda situación: Andrea observa que un grupo de niños de grado $3^{\circ}$ han salido del aula muy rápido llevando una pelota, se obtienen los siguientes resultados:

Posibles acciones: ¿Qué posibles acciones podrían realizar los niños?

Análisis: un 69\% responden "jugar algún deporte". De las acciones realizadas, menos de la mitad del grupo coincidieron en responder otro tipo de actividades como: romper vidrios, golpearse con la pelota, jugar ponchados, etc.

III. Del análisis de las gráficas se obtuvieron las siguientes respuestas a las preguntas del numeral 1 al 5: 
Gráfico 2

Nivel №2 "Comparativo" - P. Inicial

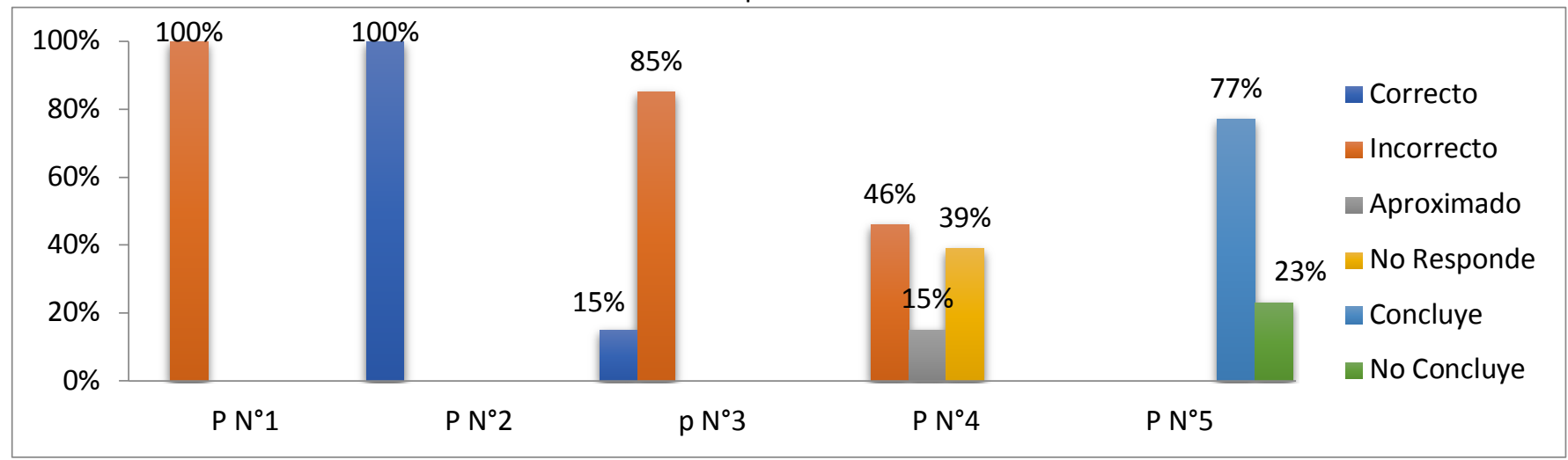

Fuente: elaboración propia

Análisis: con respecto al nivel 2: comparativo, se puede afirmar que la mayor parte de los estudiantes logran interpretar e integrar datos en el gráfico; pero presentan un poco de dificultad al comparar cantidades, aplicar conceptos matemáticos (media aritmética) y realizar conclusiones de las mismas.

IV. Cálculo de Probabilidades: dada la actividad planteada con la ruleta de colores se obtuvieron los siguientes resultados, al calcular las posibilidades de que sucedan ciertas situaciones: salga color rojo (25\%), salga color amarillo (25\%), salga color verde (25\%), salga color azul (25\%).

Análisis: en su totalidad, el grupo de estudiantes logra determinar la posibilidad de que suceda un evento, sin embargo, no tienen definido el concepto de probabilidad.

Caso menos probable: ¿Qué color es menos probable que salga al girar la ruleta? ¿Por qué?

Análisis: todos los estudiantes logran determinar el caso menos probable de una serie de eventos.

V. Con base en la situación planteada de representar gráficamente las variables presentes en la pregunta ¿Cuál es el tipo de ritmo musical que más escuchas en el día? Y teniendo en cuenta que los encuestados han brindado un ritmo entre semana y otro de fin de semana se obtuvieron los siguientes resultados:

Gráfico 3

Nivel №3 "Predictivo" - P. Inicial

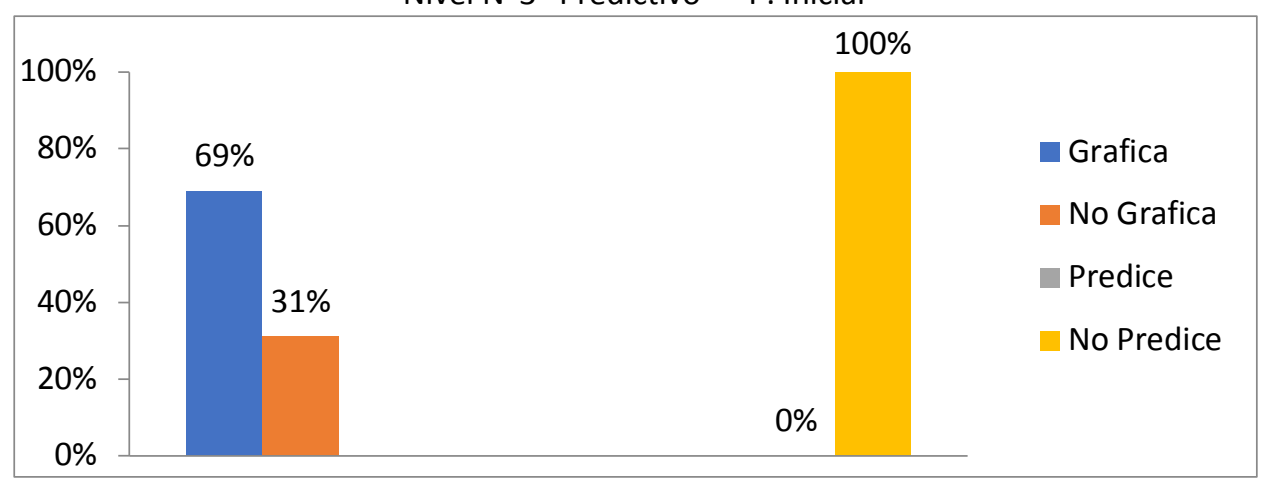

Fuente: elaboración propia

Análisis: más de la mitad del grupo de estudiantes representan en gráficos adecuados la información presentada en tablas, pero presentan dificultad en complementar información del gráfico tales como el título y variables.

Falencias. Se tomaron como base para diseñar y enfocar las actividades basadas en situaciones problema del contexto: 
- Dificultad al comparar cantidades, aplicar conceptos matemáticos (media aritmética) y realizar conclusiones de las mismas; con base en lo anterior, se evidencia que los estudiantes no alcanzan a superar el nivel 2: Comparativo.

- Se les dificulta realizar gráfica de barras dobles agrupadas para representar las variables cualitativas que se están comparando.

- Al representar datos en cualquier tipo de gráfico, ignoran información como títulos y variables.

- No logran realizar predicciones e inferencias a partir de los datos sobre informaciones que no se reflejan directamente en el gráfico "leer más allá de los datos", con lo que se concluye que los estudiantes no alcanzan a llegar al nivel 3: Predictivo.

Fortalezas. Se resaltan las siguientes:

- Los estudiantes logran identificar los datos representados en los gráficos, lo que indica que superan satisfactoriamente el nivel 1: Lectura literal.

- Logran representar datos estadísticos según la variable dada en el gráfico más adecuado.

- Interpretan la información no explícita contenida en una tabla simple y en un gráfico de líneas.

- Determinan el valor numérico de la probabilidad correspondiente a cada suceso, utilizando fracciones y explican si un suceso tiene mayor probabilidad de ocurrir que otro realizando comparaciones.

- Identifican a partir del tema de estudio y las preguntas planteadas, el tipo de variables estadísticas involucradas en la investigación y explican sus respuestas apoyándose en las características de la variable.

\subsection{Análisis de las actividades}

\section{Actividad №1. ¿Qué materia van perdiendo los alumnos de mi grado?}

Se inicia socializando con el grupo de estudiantes conceptos básicos como: población, muestra y variable por medio de un mapa conceptual; se parte de ejemplos relacionados con el contexto.

Al clasificar las variables y dar un ejemplo claro de una de ellas, los estudiantes proponen más ejemplos, esta vez relacionados con aspectos de su hogar o entorno familiar, para finalmente socializar los ejemplos planteados.

Análisis: al culminar la primera etapa de la actividad, se evidencia que más de la mitad del grupo logra plantear ejemplos coherentes relacionados con su contexto y son muy pocos los que se les dificulta proponer ejemplos.

Continuando con la segunda etapa de la actividad donde se trabaja con dos tipos de variable: cuantitativa y cualitativa respectivamente, los estudiantes inician la primera parte de esta etapa recolectando información sobre la materia en la que obtuvieron más baja nota en el tercer periodo. Para obtener la información de una manera ordenada y rápida, se selecciona un estudiante para que realice la pregunta a cada uno de los compañeros y anote las respuestas en el tablero.

Teniendo la información en el tablero, cada estudiante inicia a organizarla en una tabla de frecuencias para luego representar dichos datos en tres tipos de gráficos: pictograma, diagrama de barras y diagrama circular.

Basándose en la información registrada en los gráficos, los estudiantes dan respuesta a las preguntas planteadas, coincidiendo la mayor parte del grupo en sus respuestas.

- ¿Qué tipo de variable son las materias? Rta: son variables cualitativas

- ¿Cuáles son las materias en las que obtuvieron la más baja nota? Rta: física, geometría, ética, informática y sociales.

- ¿ ¿Tendrá sentido calcular el promedio de estas materias? Rta: No

- ¿ ¿En qué materia se registra mayor porcentaje de pérdida? Rta: Física 
- ¿Cuántos estudiantes no perdieron ninguna materia en el tercer periodo? Rta: 11 estudiantes

- ¿ ¿Qué porcentaje de estudiantes están perdiendo alguna materia en el tercer periodo? Rta: el 58\% está perdiendo alguna materia.

De las respuestas obtenidas por los estudiantes se llega a las siguientes conclusiones:

- En su totalidad el grupo de estudiantes logra clasificar la variable a la cual corresponde las materias.

- Son muy pocos los estudiantes que logran dar una razón justificada del porqué no se puede calcular el promedio de las materias.

- La mayoría del grupo presenta dificultad al hallar el porcentaje de estudiantes que están perdiendo alguna materia, pero con una breve orientación, logran dar la respuesta correcta a la pregunta planteada.

Para continuar con la segunda parte de la actividad, se plantea ahora la pregunta ¿Cuál fue la nota más baja que obtuvo en el tercer periodo? siguiendo la misma estrategia que en el punto anterior en la recolección de los datos.

Teniendo la información registrada en el tablero, los estudiantes elaboran la tabla de frecuencias, esta vez, creando intervalos ya que los datos se deben agrupar; se continúa con la representación de los datos obtenidos en dos tipos de gráficos: histograma y diagrama de frecuencias.

Con base en las gráficas realizadas, y algunos ejemplos orientados de cómo analizar una gráfica, los estudiantes logran llegar a las siguientes conclusiones:

- Solo un estudiante del grupo obtuvo una nota entre 40 y 45.

- La mitad de los estudiantes de grado 8-2 están perdiendo una materia con notas menores a 30 y mayores que 20.

- El $19 \%$ de los estudiantes están perdiendo una materia con nota entre 20 y 25.

- Ningún estudiante obtuvo una nota inferior a 20.

Análisis: a la pregunta propuesta ¿Podríamos dar un valor promedio de las notas obtenidas en todo el grupo? el total de los estudiantes coincide en responder que sí; a diferencia de la pregunta similar propuesta en la actividad anterior, los estudiantes logran dar una razón justificada y hallar el promedio de las notas más bajas en el grupo.

\section{Actividad №2 “Conozco mejor a mis compañeros"}

Para dar inicio a la primera parte de la actividad, se toman como base 90 encuestas, las cuales fueron contestadas previamente por los estudiantes de los demás octavos (8-1, 8-3 y 8-4); se organizan cuatro grupos de cuatro estudiantes y dos grupos de cinco estudiantes; se hace entrega por grupo de 15 encuestas. Los estudiantes inician a organizar la información de las 21 preguntas planteadas en su cuaderno, para luego ser compartida a los demás grupos.Teniendo la información organizada, se asignó a cada grupo una cantidad de preguntas de la encuesta (en promedio se asignaron cuatro preguntas), donde unificaron la información obtenida en los demás grupos por medio del software Excel, utilizando como herramienta tecnológica el celular o computadora.

Como segunda parte de la actividad, los estudiantes en grupo representaron los datos obtenidos en el mejor gráfico según la variable correspondiente a las preguntas asignadas y realizaron análisis y conclusiones.

Para culminar con la actividad, se socializó el trabajo realizado en cada grupo y se tomaron notas de las conclusiones a las cuales llegaron los estudiantes frente a la situación problema planteada al inicio de la actividad: factores que influyen en el bajo rendimiento de los estudiantes.

El material elaborado por los estudiantes (gráficas, análisis y conclusiones), se organizó en un solo informe y se entregó al coordinador académico como soporte para la Institución. 
Análisis: en esta última parte de la actividad, se evidenció que la mayoría del grupo presentó dificultad cuando comenzaron a representar los datos, debido a que no recordaban cómo graficar en Excel; esta dificultad fue superada con una breve orientación.

Todos los grupos alcanzaron a terminar con éxito la actividad planteada y llegaron a las siguientes conclusiones acerca de los factores que influyen en el bajo rendimiento de algunos estudiantes:

- El nivel educativo de los padres puede ser un factor que influya en el bajo rendimiento de los estudiantes, ya que no pueden orientarlos en sus tareas escolares. Esto basado en que más de la mitad de los padres de familia de los estudiantes encuestados, poseen estudios hasta educación básica. Pero mayor aún se hace énfasis en el nivel escolar de la madre, ya que es ella quien pasa más tiempo con sus hijos. Si la mamá posee una preparación académica, puede apoyar a sus hijos en las tareas escolares e incluso reforzar conocimientos aprendidos en clases.

- El ingreso económico de la familia, es otro factor que influye en el bajo rendimiento académico, según los resultados obtenidos en la encuesta, más de la mitad de los hogares de los estudiantes reciben un ingreso mensual entre 1 y 2 SMLV, esto no permite que el dinero alcance en el hogar para comprar todos los implementos necesarios para su aprendizaje y más aún cuando son varios hijos en edad escolar; esto también genera que los estudiantes se vean obligados a trabajar y estudiar al mismo tiempo.

- Aunque solo el $11 \%$ de los estudiantes encuestados viven en zona rural, esto es un aspecto que influye en el bajo rendimiento académico, ya que en sus casas no cuentan con servicio de internet; no pueden quedarse después de las clases a realizar sus tareas, ya que la ruta escolar parte tan pronto salgan los estudiantes y peor aún, el colegio no cuenta con restaurante escolar, lo cual generaría un gasto adicional.

- Según los resultados obtenidos el $90 \%$ de los estudiantes se siente conforme y feliz en la Institución Educativa donde estudian, el otro $10 \%$ justifican que estudian en la institución por castigo de sus padres. Para Robbins (2004) la motivación "es un proceso que da cuenta de la intensidad, dirección y persistencia del esfuerzo por conseguir una meta" (p. 156). Basado en lo anterior, cuando un estudiante no está suficientemente motivado, se ve reflejado en su rendimiento académico.

\subsection{Análisis de la Prueba diagnóstica - Final}

I. De las preguntas planteadas del numeral 1 al 6 , se obtuvieron los siguientes resultados:

Gráfico 4

Nivel №1 "Lectura literal" - P. Final

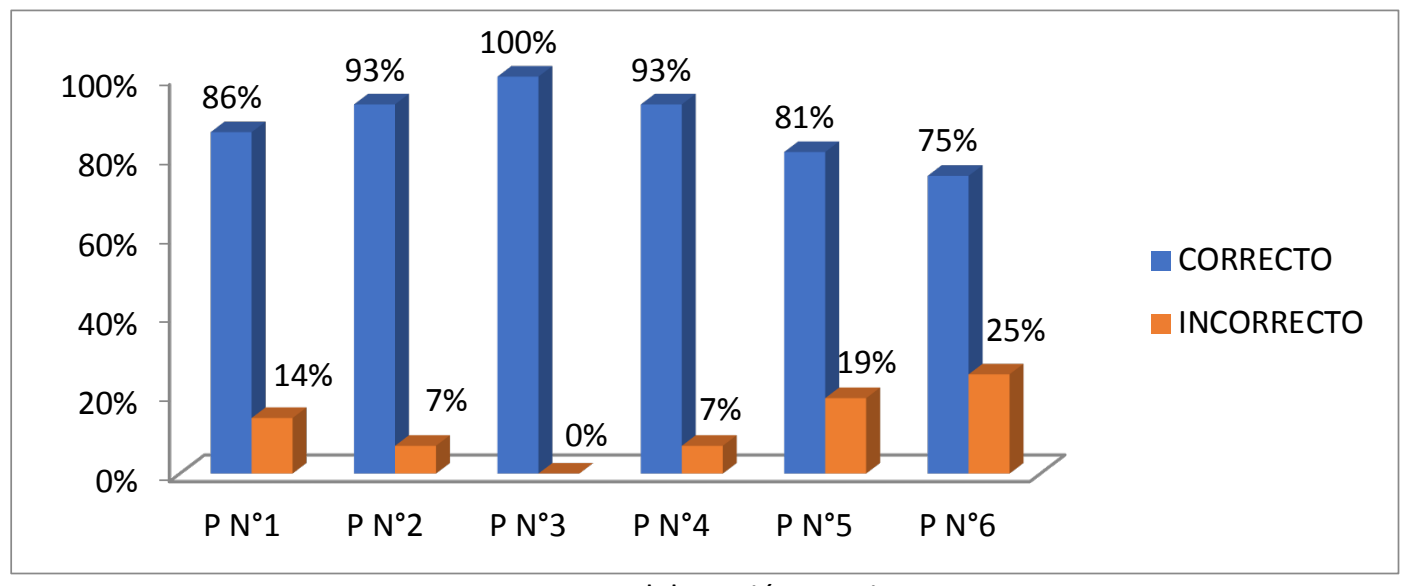

Fuente: elaboración propia

Análisis: la mayoría del grupo de estudiantes logra superar satisfactoriamente el primer nivel de lectura literal de gráficos, es decir, leen los datos del gráfico sin necesidad de realizar una interpretación de la información contenida en el mismo. 
II. Del análisis de las gráficas se obtuvieron las siguientes respuestas a las preguntas del numeral 1 al 5 :

Gráfico 5

Nivel №2 "Comparativo" - P. Final

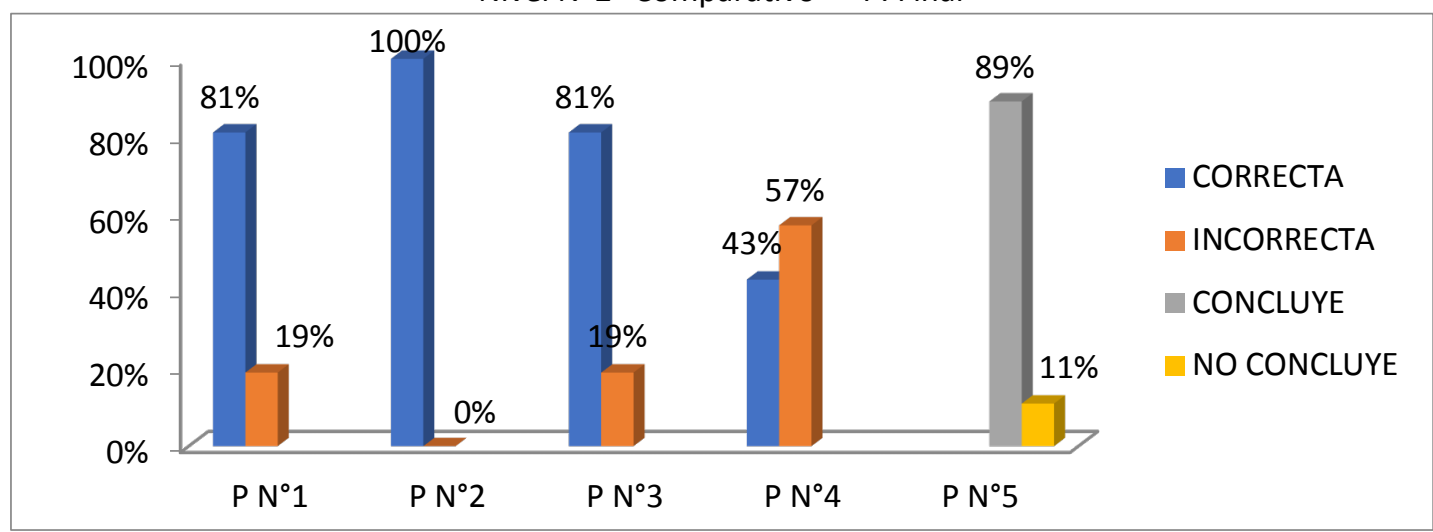

Fuente: elaboración propia

Análisis: la mayor parte del grupo de estudiantes interpretan e integran datos en el gráfico; además de esto logran comparar cantidades, aplicar conceptos matemáticos (media aritmética) y realizar conclusiones de las mismas. Con lo que se evidencia que logran superar satisfactoriamente el nivel 2.

III. Con base en el estudio planteado sobre el tiempo que intervine un adolescente en el uso del celular como entretenimiento durante los fines de semana y teniendo en cuenta las variables que intervienen para este estudio, se obtuvieron los siguientes resultados:

Gráfico 6

Nivel №3 “Predictivo" - P. Final

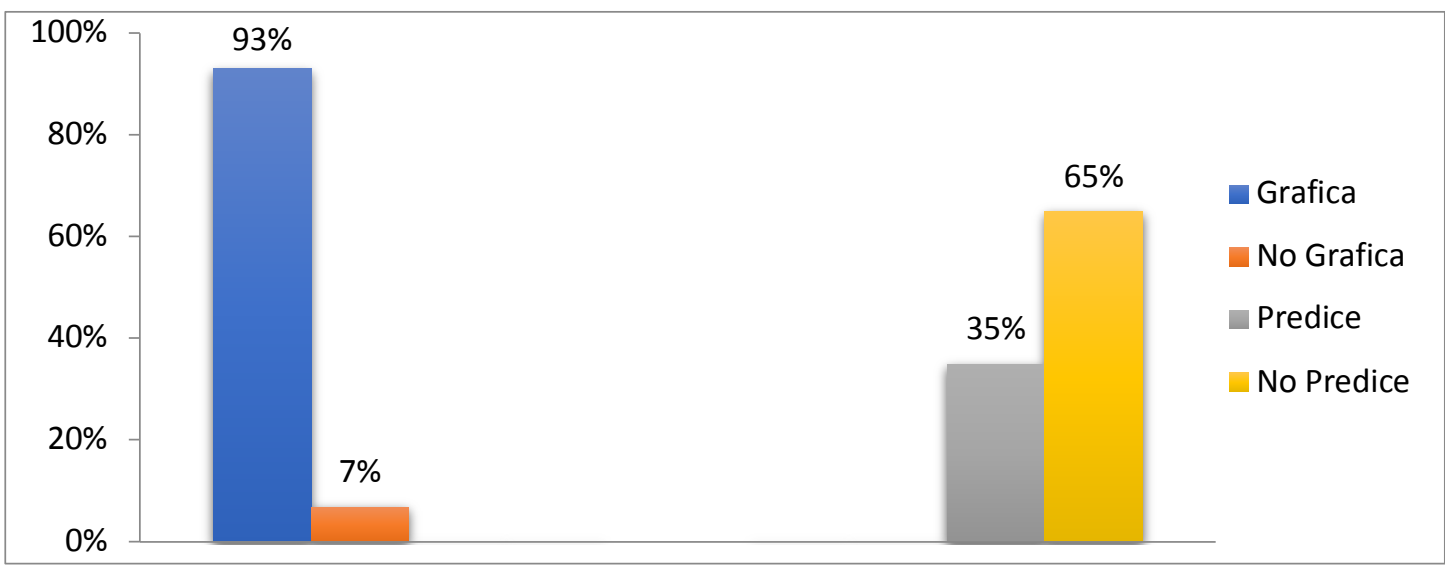

Fuente: elaboración propia

Análisis: casi la totalidad del grupo de estudiantes representa en gráficos adecuados la información presentada en tablas, ubicando la información del título y variables; menos de la mitad del grupo de estudiantes logran realizar predicciones e inferencias sobre informaciones que no se reflejan directamente en el gráfico "leer más allá de los datos"

IV. Dada la situación problema del contexto, donde el estudiante debía actuar como investigador activo, se obtuvieron los siguientes resultados: 
Gráfico 7

Nivel №4 “Integrativo" - P. Final

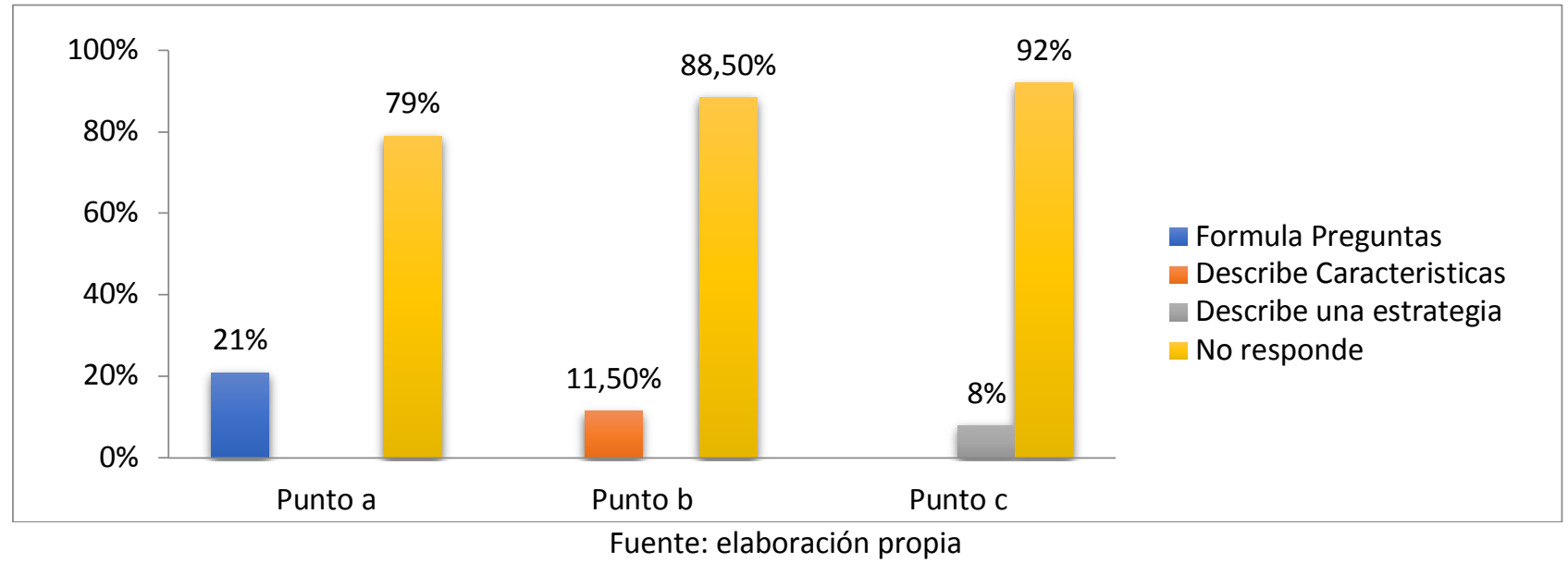

Análisis: solo un mínimo del grupo de estudiantes logra formular una pregunta de investigación dada una situación problema del contexto. La mayor parte del grupo no responde a las preguntas planteadas con lo cual queda en evidencia que solo logran ser receptores de información.

\section{Falencias:}

- No logran formular una pregunta de investigación dada una situación problema del contexto; solo logran ser receptores de información.

- No logran realizar predicciones e inferencias sobre informaciones que no se reflejan directamente en el gráfico "leer más allá de los datos"

\section{Fortalezas:}

- Interpretan e integran datos en el gráfico; además logran comparar cantidades, aplicar conceptos matemáticos (media aritmética) y realizar conclusiones. Con lo que se evidencia que logran superar satisfactoriamente el nivel 2: Comparativo.

- Representan la información presentada en gráficos y tablas, ubicando adecuadamente la información del título y variables.

\subsection{Comparación de resultados de la prueba diagnóstica inicial y final}

Se realiza el análisis de acuerdo a los niveles establecidos en la fase 2 (véase tabla 4) y utilizando el programa estadístico informático SPSS.

\section{Nivel 1: Lectura literal (ver Gráfico 8)}

Análisis: al observar las "estadisticas de muestras emparejadas" y la "prueba de muestras emparejadas" se obtiene $p=0.180>0.05$ (nivel de significancia), lo cual indica que no existen diferencias significativas entre las medias de la prueba diagnóstica inicial y final, con respecto al nivel 1. 
Gráfico 8

Comparación de resultados prueba inicial y final. Nivel 1: Lectura literal

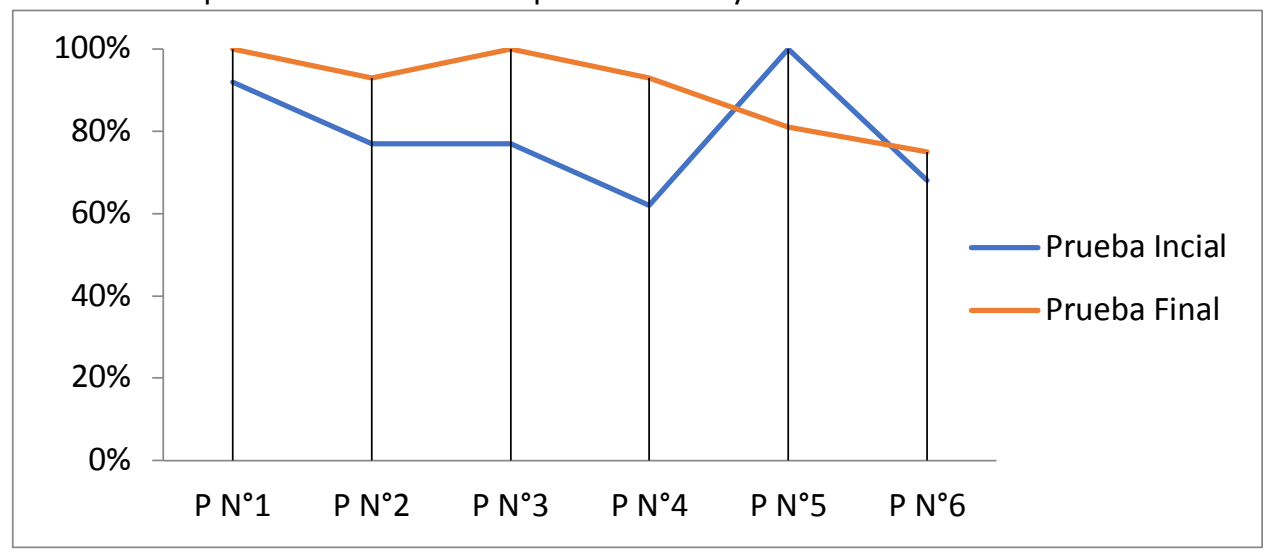

Fuente: elaboración propia

\section{Nivel 2: Comparativo (ver Gráfico 9)}

Análisis: al observar las "estadisticas de muestras emparejadas" y la "prueba de muestras emparejadas" se obtiene $p=0.059>0.05$ (nivel de significancia) lo cual indica que no existen diferencias significativas entre las medias de la prueba diagnóstica inicial y final, con respecto al nivel 2.

Gráfico 9

Comparación de resultados prueba inicial y final. Nivel 2: Comparativo

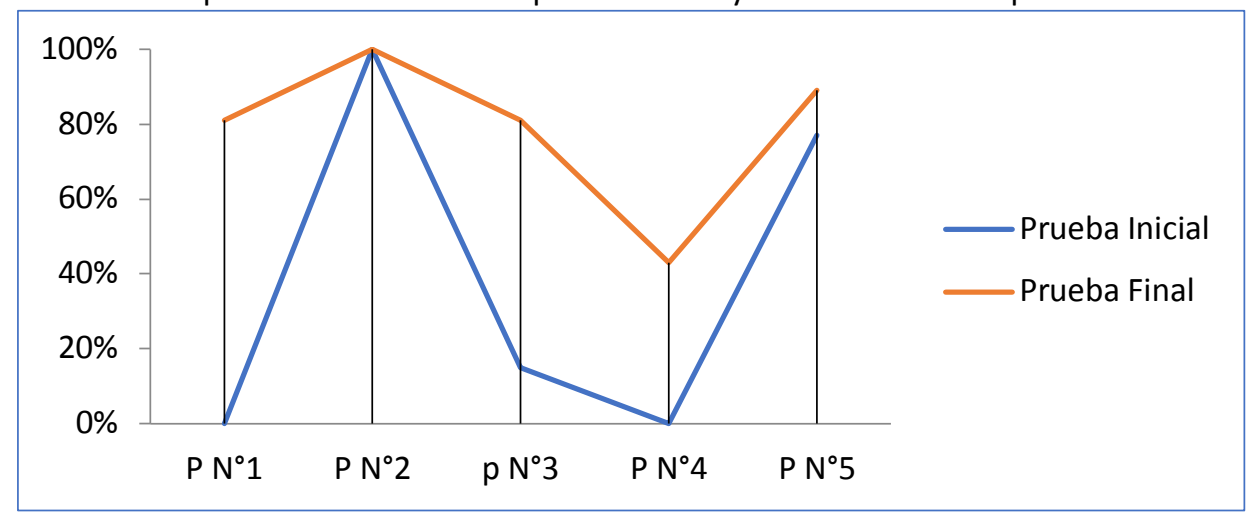

Fuente: elaboración propia

\section{Nivel 3: Predictivo (ver Gráfico 10)}

Análisis: al observar las "estadisticas de muestras emparejadas" y la "prueba de muestras emparejadas" se obtiene $p=0.117>0.05$ (nivel de significancia) lo cual indica que no existen diferencias significativas entre las medias de la prueba diagnóstica inicial y final, con respecto al nivel 3. 
Gráfico 10

Comparación de resultados prueba inicial y final. Nivel 3: Predictivo

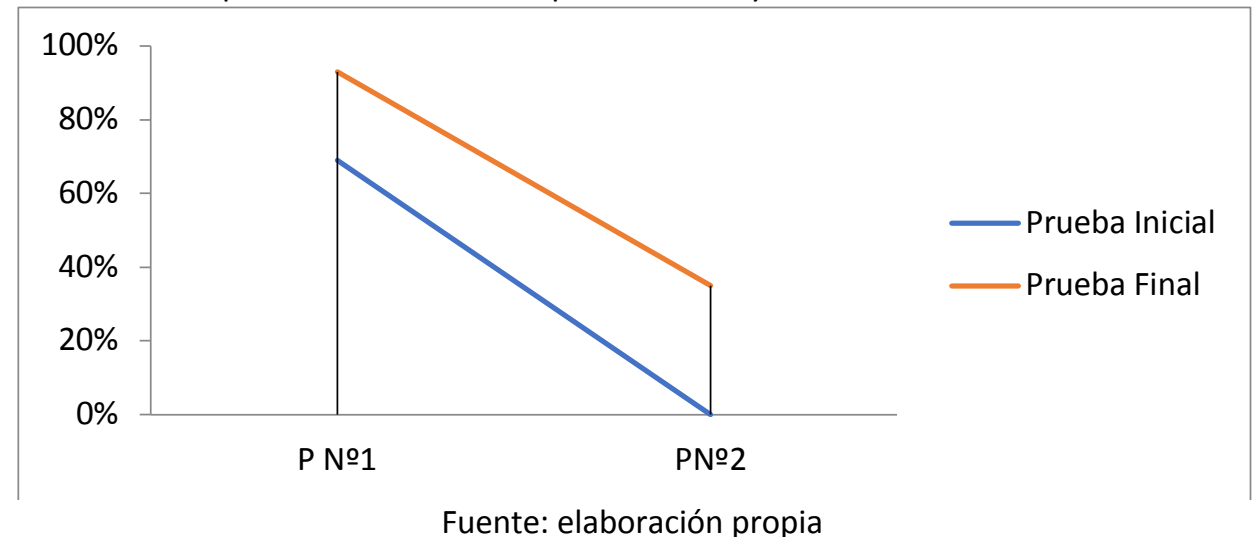

\section{Conclusiones}

La comparación de los resultados de la prueba diagnóstica inicial y final, arrojaron que no hubo diferencias significativas, sin embargo, sí hubo un aumento en el porcentaje de estudiantes que contestaron correctamente las preguntas planteadas en los tres niveles.

Con base en los niveles tomados como referencia en la estructuración de las pruebas diagnósticas (Nivel 1: Literal, Nivel 2: Comparativo, Nivel 3: Predictivo y Nivel 4: Integrativo) se evidenció en la prueba diagnóstica final el fortalecimiento de aspectos como: la adecuada comparación e interpretación de datos según las características de un gráfico, descripción de relaciones matemáticas, uso de diferentes tipos de representación, elaboración de conclusiones coherentes acordes a datos representados en tablas y gráficos; logrando así, no solo cumplir con los indicadores propuestos en algunos de los niveles ya mencionados, sino también, reforzar las formas de proceder (las competencias) y los aspectos conceptuales y estructurales de la matemática (los componentes) que tiene establecidos el ICFES al momento de evaluar en las pruebas SABER 3, 5ㅇ y 9o.

Al lograr que los estudiantes superaran satisfactoriamente los niveles 1 y 2 (Literal y Comparativo respectivamente) y representaran datos en gráficos adecuados según la variable dada en la información, se evidencia un fortalecimiento del razonamiento estadístico.

La prueba diagnóstica final, se estructuró bajo cuatro niveles que fusiona la compresión de gráficos; este último (Nivel 4: Integrativo) evidenció que los estudiantes logran fortalecer el razonamiento, más no alcanzan un desarrollo total del pensamiento estadístico.

Los estudiantes logran cumplir con las actividades propuestas de una manera eficiente y a la vez motivados, ya que buscan dar solución a una situación problema de su contexto. Con el uso de software se puede realizar un control más adecuado sobre las actividades que realizan los estudiantes, logrando también en ellos, una mejor disposición para analizar y expresar conclusiones de resultados estadísticos.

La aplicación de situaciones problema del contexto, como estrategia pedagógica en las actividades de profundización, evidenció en el grupo de estudiantes un proceso de aprendizaje, basado en el interés y la motivación, que les permitió contextualizar sus conocimientos y dar utilidad a sus diferentes saberes; logrando así, acercar el aprendizaje a la realidad de los estudiantes y como consecuencia fortaleció el razonamiento estadístico contribuyendo con el desarrollo del pensamiento estadístico dando por cierta la hipótesis planteada.

En el grupo que se tomó como muestra (8-2), se encontró que aunque el p-valor (significancia) del resultado obtenido al realizar la comparación de las dos pruebas diagnósticas aplicadas está por encima del límite $\alpha=$ 0.05, la diferencia de ambos valores es mínima, además de esto, se evidencia que la media de la prueba 
diagnóstica final aumenta con respecto a la inicial. Con base en lo anterior, se puede deducir que aunque no hubo un avance significativo, sí se evidenció un cambio cognitivo y una variación en el rendimiento académico. Lo que demuestra que la aplicación de actividades basadas en situaciones problema del contexto y el uso del software Excel fortaleció el razonamiento y contribuyó al desarrollo del pensamiento estadístico.

Este proyecto de investigación se socializó con docentes del área de matemáticas del IETD de Monterrey, el cual tuvo gran aceptación por la metodología aplicada y los resultados obtenidos en el grupo muestra (8-2); llegando así a la conclusión de que se implementaría en los demás grupos en los años académicos posteriores como proyecto transversal del área y como base para corroborar los resultados obtenidos en la aplicación de las futuras prueba SABER 3, 5ㅇ y 9ㅇ.

\section{Referencias}

Aoyama, K. (2007). Investigating a hierarchy of students' interpretations of graphs. International Electronic Journal of Mathematics Education, 2(3), 298-318.

Batanero, C., \& Godino, J. D. (2002). Estocástica y su didáctica para maestros. Departamento de Didáctica de la Matemática, Universidad de Granada.

Chick, H. L., \& Watson, J. M. (2002). Collaborative influences on emergent statistical thinking - a case study. The Journal of Mathematical Behavior, 21(3), 371-400.

Curcio, F. R. (1989). Developing graph comprehension. Reston, VA: NCTM.

Friel, S., Curcio, F. \& Bright, G. (2001). Making sense of graphs: critical factors influencing comprehension and instructional implications. Journal for Research in mathematics Education, 32(2), 124-158.

Gal, I. (2002). Adults' statistical literacy: Meanings, components, responsibilities. International statistical review, 70(1), 1-25.

Galli, R. (2015). Principios y algoritmos de concurrencia. Ricardo Galli.

Garcia, J., Hoyos, E., \& Acosta, C.(2014). El desarrollo del pensamiento estadístico en niños y niñas del grado $7^{\circ}$ de básica secundaria, una estrategiaguiada por software educativo. (Tesis de maestria). Universidad del Quindío. Colombia.

Garfield, J., \& Ben-Zvi, D. (2008). Developing students' statistical reasoning: Connecting research and teaching practice. Springer Science \& Business Media.

Gómez, B. R. (2003). Aportes de la investigación-acción educativa a la hipótesis del maestro investigador: evidencias y obstáculos. Educación y educadores, (6), 91-104.

González, W. (2018). Resolución de Problemas, Una Estrategia para Aprender Estadística. (Tesis de maestria). Universidad Externado de Colombia, Bogotá D. C.

Hernández, S. (2006). Metodología de la investigación. McGraw-Hill. Cuarta edición

Jimenez Ramirez, J. V., \& Inzunsa Cazares, S. (2011). Razonamiento y pensamiento estadístico en estudiantes universitarios. In XIII Conferência Interamericana de Educação Matemática. Brasil: Recife.

MEN (1998). Lineamientos curriculares en Matemáticas. Ministerio de Educación Nacional. Colombia.

MEN (2006). Estándares Curriculares de Matemáticas. Ministerio de Educación Nacional. Colombia.

NCTM. (2000). Principles and standards for schools mathematics. Reston, VA: NCTM. 
Obando, G. \& Muñera, J. (2003). Las situaciones problema como estrategia para la conceptualización matemática. Revista educación y pedagogía, 15(35).

Osorio, M., Suárez, A., \& Uribe, C. (2011). Revisión de aspectos asociados a la problemática del aprendizaje de la Probabilidad. Revista Virtual Universidad Católica del Norte, 34(34), 360-384.

Palacios, A., \& Tafur, G. (2018). La resolución de problemas como estrategia de enseñanza para el desarrollo del pensamiento aleatorio. Un estudio realizado con estudiantes de grado quinto y sexto de dos instituciones de Yopal- Casanare. (Tesis de Maestría). Universidad de La Salle. Colombia.

Ramirez, L. (2017). Fortalecimiento del Pensamiento Aleatorio en Estudiantes de Grado Tercero de la Institucion Educativa Académico de Guadalajara de Buga en el Segundo Semestre de 2016. (Tesis de maestria). Universidad del Cauca. Colombia.

Robbins, S. P. (2004). Comportamiento organizacional. Pearson educación.

Sánchez, E., \& Gómez-Blancarte, A. L. (2011). El desarrollo del pensamiento estadístico de profesores de secundaria en servicio. Investigaciones actuales en educación estadística y formación de profesores, 55-72.

Wild, C., \& Pfannkuch, M. (1999). Statistical thinking in empirical enquiry. International Statistical Review, 67(3), 223-265.

Zapata, L., \& González, D. (2017). Imágenes de los profesores sobre la estadística y su enseñanza. Educación matemática, 29(1), 61-90.

Zapata, L., \& Rocha, P. (2016). Teachers' questions in the statistics class. In The Teaching and Learning of Statistics (pp. 271-278). Springer, Cham.

Esta obra está bajo una Licencia Creative Commons Attribución-NoCommercial 4.0 International

(cc) BY-NC 